\title{
High Resolution Electron Microscope Observation of Medium Range Atomic Ordering in an Amorphous Pd-Si Alloy
}

\author{
By Yoshihiko Hirotsu*, Kazunori Anazawa** and Tadakatsu Okubo**
}

\begin{abstract}
High resolution electron microscope images in amorphous $\mathrm{Pd}-\mathrm{Si}$ alloy were calculated with various structure models and compared with the observed ones. For the structure models, atomic models of (1) dense random packing (DRP), (2) FCC atom clusters (embedded in DRP structure) and (3) icosahedral atom clusters (embedded in DRP structure) were constructed by using the Lennard-Jones type atomic potentials. Structure observations on the atomic level were made with a high resolution electron microscope at $200 \mathrm{kV}$ for amorphous $\mathrm{Pd}_{80} \mathrm{Si}_{20}$ thin films deposited at $195 \mathrm{~K}$ by beam-sputtering technique. Atom clusters as large as 1-2 nm having the FCC-Pd type structure were observed locally. These FCC clusters are embedded in the structure which is thought to be that of DRP. A radial distribution function (RDF) calculated for the FCC cluster in the DRP structure was found to explain well the experimental RDF obtained by other reseachers for the amorphous $\mathrm{Pd}_{80} \mathrm{Si}_{20}$ alloy.
\end{abstract}

(Received April 2, 1990)

Keywords: high resolution electron microscopy, amorphous alloy structures, medium range ordering, palladium-silicon alloy, face-centered cubic atom cluster

\section{Introduction}

Recent structural studies by high-resolution electron microscopy $(\text { HREM })^{(1)-(5)}$ in amorphous alloys have revealed the formation of local microcrystalline domains extending in a range as large as $1 \sim 2 \mathrm{~nm}$. The medium range ordering (MRO) $)^{(6)(7)}$ of atoms, which is supposed to be formed in the dense random packing (DRP) structure, is now becoming an important concept in understanding the structure of amorphous alloys. In our previous HREM studies on amorphous $\mathrm{Fe}_{84} \mathrm{~B}_{16}{ }^{\left({ }^{(3)}\right.}$ and $\mathrm{Pd}_{77.5} \mathrm{Cu}_{6} \mathrm{Si}_{16.5}$ alloys ${ }^{(4)(5)}$ prepared by the liquid-quenching (roll-quenching) technique, MRO regions with crossed lattice images were observed. Based on the spacings and geometies of these crossed lattice images, the structures of the MRO domains were identified as BCC and FCClike atom clusters in the $\mathrm{Fe}_{84} \mathrm{~B}_{16}$ and $\mathrm{Pd}_{77.5} \mathrm{Cu}_{6} \mathrm{Si}_{16.5}$ amorphous alloys, respectively. In these studies, the observation frequency of the MRO domains was found to be dependent on such liquid-quenching conditions as the roll-speed and the melt temperature.

In the HREM study of amorphous alloys, liquidquenched amorphous alloy ribbons have been used as specimens. Technically, there is a limit to increase the quenching rate in the case of liquid-quenching. On the other hand, vapor-quenching techniques, such as vacuum evaporation and beam sputtering, have an advantage in getting higher quenching rate than the liquidquenching techniques. To investigate the MRO structure of amorphous alloys, it is important to compare the struc-

* Department of Mechanical Engineering, Nagaoka University of Technology, Kamitomioka-cho, Nagaoka, 940-21, Japan.

** Graduate Student, Nagaoka University of Technology, Nagaoka. tures formed under different amorphization conditions. The vapor-quenching techniques are advantageous not only in getting higher quenching rates, but also in obtaining specimens suitable for the HREM observation. For the liquid-quenched amorphous ribbon specimens, great cares must always be taken in thinning the specimens for the observation to avoid a specimen contamination and oxidation, or occasionally a local specimen heating. In the case of vapor-quenched specimens, on the other hand, such cares are not necessary to be taken. In addition to it, the specimen (film) thickness can be controlled suitably for the HREM observation. For preparing good amorphous thin film, it is necessary to deposit films onto low temperature substrates in a high vacuum.

In order to examine HREM images of amorphous alloys for studying the MRO structure, detailed HREM image simulations have to be made based on possible structure models. Recent computer simulation studies of amorphous structures ${ }^{(8)-(10)}$ have suggested a possible formation of MRO with icosahedral atomic clusters. An amorphous structure without MRO is possible when the quenching rate for amorphization is quite high. Such a structure has been observed by HREM specially in intermetallic compounds when they were irradiated by fast electrons at low temperature ${ }^{(11)}$. For amorphous alloys like $\mathrm{Pd}-\mathrm{Si}$ or $\mathrm{Fe}-\mathrm{B}$ alloys, it is, therefore, meaningful to calculate HREM images not only of the MRO structures with FCC or BCC atom clusters, but also of the icosahedral MRO structure and also the DRP structure and compare them with observed images. Such studies have not been performed intensively. In the structure modeling, it must be taken into account that possible models are able to explain not only observed HREM images but also X-ray or neutron scattering intensities or radial distribution functions (RDF) from the amorphous 
alloys concerned.

In the present study, atomic models of (1) DRP structure, (2) FCC MRO structure (one FCC cluster embedded in the DRP structure) and (3) icosahedral MRO structure (one icosahedral cluster embedded in the DRP structure) were constructed in computer for an amorphous $\mathrm{Pd}-\mathrm{Si}$ alloy based on the energy minimization of the atomic configurational energy. The HREM images for these models were then calculated under various defocus conditions and observation directions. The calculated images were compared with those taken from amorphous $\mathrm{Pd}_{80} \mathrm{Si}_{20}$ alloy thin films prepared by Ar beam-sputtering. Amorphous structures in the present $\mathrm{Pd}_{80} \mathrm{Si}_{20}$ alloy film and those in the $\mathrm{Pd}_{77.5} \mathrm{Cu}_{6} \mathrm{Si}_{16.5}$ alloy ribbons, which we previously investigated, were compared and discussed in relation to the quenching conditions.

\section{Structure Models and Image Calculations}

Atomic DRP, MRO and icosahedral cluster models were made with a personal computer by assuming a $\mathrm{Pd}_{80} \mathrm{Si}_{20}$ alloy near the eutectic composition. In the structure modeling for the DRP model, Pd and Si atoms (total 600 atoms) in $\mathrm{Pd}_{80} \mathrm{Si}_{20}$ having a density of 0.67 were first distributed randomly. By introducing the Lennard-Jones type atomic potentials $\phi\left(r_{i j}\right)$ for atoms $\mathrm{i}$ and $\mathrm{j}$ separated by $r_{i j}$, total atomic configurational energies were then calculated and were minimized by the iteration procedure (iteration: 200 times or more). The structures of FCC and icosahedral clusters were formed from 106 and 147 atoms, respectively, by distributing $\mathrm{Pd}$ and $\mathrm{Si}$ atoms randomly on their specified positions and by confining the composition to $\mathrm{Pd}_{80} \mathrm{Si}_{20}$. These clusters were enveloped in the $\mathrm{Pd}_{80} \mathrm{Si}_{20}$ DRP structure followed by the structure relaxations as mentioned above. The enveloping DRP structure must be necessary especially when the quenching rate is high for the amorphization. The form of the interatomic potential is

$$
\phi\left(r_{i j}\right)=4 \varepsilon\left\{\left(\sigma / r_{i j}\right)^{12}-\left(\sigma / r_{i j}\right)^{6}\right\} .
$$

The parameter $\varepsilon$ and the equilibrium distance $R$ for each atom pairs used in the structural study of $\mathrm{Pd}_{80} \mathrm{Si}_{20}$ alloy by Dubois et $a l .^{(12)}$ are listed in Table 1 . The $\sigma$ value can be derived by the equation $\sigma=(1 / 2)^{1 / 6} R$. These parameters are also used in this study.

Since a qualitative discussion is necessary at present on the MRO structure, the HREM image calculations for these DRP and MRO structure models were made based on the thin-phase object imaging theory applied previous$1 y^{(3)-(5)}$. An adaptability of the imaging theory has already been checked by calculating image intensities (one-dimensional calculation) from a FCC microcrystal with $144 \mathrm{Pd}$ atoms embedded in a DRP structure. The electron optical parameters used in the present calculation are listed in Table 2. Cubic superlattice-cell was used in the calculation with a cell size of $2.8 \mathrm{~nm}$.

Figure 1(a) to (f) are calculated examples of the through-focus images (underfocus) for the DRP structure. Images characteristic of the DRP structure are ob-
Table 1 Parameters of Lennard-Jones atomic potentials for $\mathrm{Pd}-\mathrm{Si}$ amorphous alloy $^{(12)}$.

\begin{tabular}{llll}
\hline \hline$\varepsilon$ (arbitrary unit) & \multicolumn{2}{c}{$R(\mathrm{~nm})$} \\
\hline$\varepsilon(\mathrm{Pd}-\mathrm{Pd})$ & 1.00 & $R(\mathrm{Pd}-\mathrm{Pd})$ & 0.276 \\
$\varepsilon(\mathrm{Si}-\mathrm{Si})$ & 0.72 & $R(\mathrm{Si}-\mathrm{Si})$ & 0.368 \\
$\varepsilon(\mathrm{Pd}-\mathrm{Si})$ & 0.29 & $R(\mathrm{Pd}-\mathrm{Si})$ & 0.240 \\
\hline
\end{tabular}

Table 2 Electron optical parameters used in the image calculation.

\begin{tabular}{ll}
\hline \hline Wavelength $(200 \mathrm{kV})$ & $0.00251 \mathrm{~nm}$ \\
Spherical aberration constant & $0.5 \mathrm{~mm}$ \\
Defocus due to chromatic aberration & $5.0 \mathrm{~nm}$ \\
Beam divergence angle & $1.0 \times 10^{-4} \mathrm{rad}$ \\
\hline \hline
\end{tabular}

served finely in relatively high contrasts under the defocus $(\Delta f)$ from about 60 to $80 \mathrm{~nm}$. It is noted that pseudo-circular bright contrasts as indicated in Fig. 1(c) by arrows are frequently seen in the calculated DRP images. The atomic structures producing such pseudo-circular image was examined by removing atoms enveloping the structure and by recalculating the images. After several examinations of such images calculated from different DRP structures, it was found that the pseudocircular images under the defocus come from two dimensionally arranged ring-like atomic chains with six-fold or five-fold symmetry surrounding each central atom. An example of such pseudo-circular images and the corresponding circular atomic arrangements are shown in Fig. 2(a) and (b), respectively. These pseudo-circular bright images have diameters mostly of $0.22 \sim 0.23 \mathrm{~nm}$ but sometimes have larger ones near $0.25 \sim 0.27 \mathrm{~nm}$.

An image calculation of FCC MRO structure was made in the previous study ${ }^{(3)(4)}$ without enveloping by the DRP structure. According to this study, clear lattice fringe images of atom cluster with the $\alpha-\mathrm{Pd}$ type structure appeared under the most appropriate defocus conditions reflecting the potential distribution of the atom cluster model. In the present calculation, the effect of DRP structure on the image formation of the FCC cluster was examined. Figure 3(a) to (f) are calculated images for the FCC MRO structure enveloped in 420 DRP atoms and viewed along the [101] FCC direction. It is seen that under the Scherzer focus $(\Delta f \sim 40 \mathrm{~nm})$ and also under large defocus values the MRO image is not able to be observed but is observed under the defocus condition near $\Delta f \sim 80 \mathrm{~nm}$. Figure 4(a) to (f) are calculated images for the MRO structure model but viewed along the [001] FCC direction. The crossed (200) and (020) lattice fringes with spacing of $0.19 \mathrm{~nm}$ can be observed clearly also under $\Delta f \sim 800 \mathrm{~nm}$. The DRP structure enveloping the FCC cluster was found to limit the suitable range of observation conditions and also makes the observation of the external shape of the FCC cluster difficult.

Figure 5(a) to (f) are calculated through-focus images for the icosahedral MRO model viewed along the fivefold axis. An image of projected potential with ten-fold symmetry appears along this direction when the DRP 


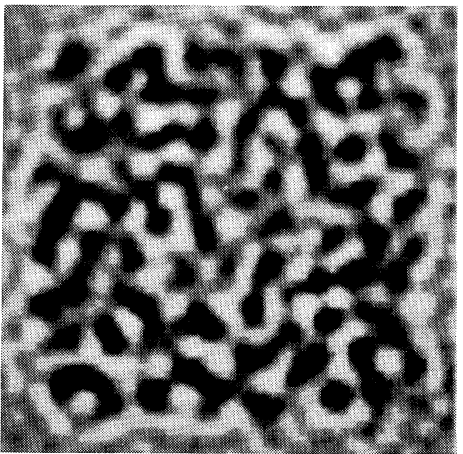

(a)

$40 \mathrm{~nm}$

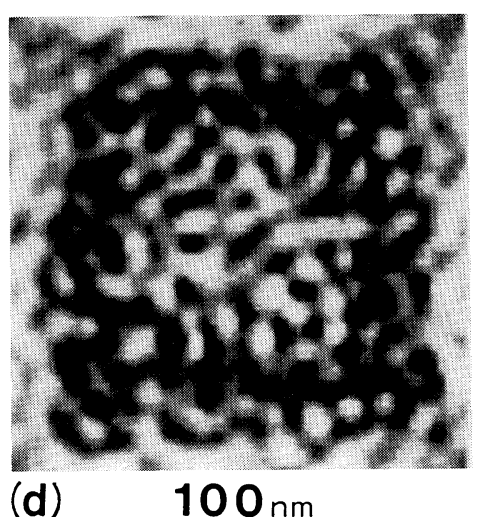

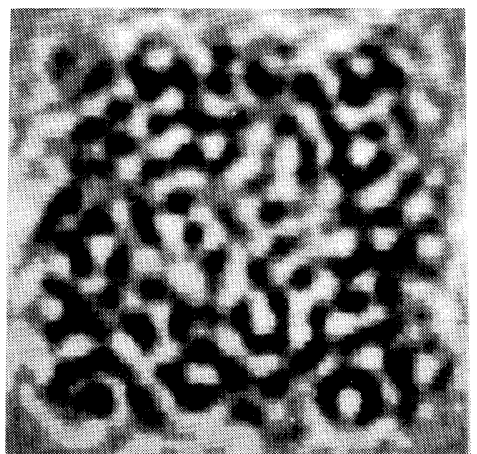

(b)

$60 \mathrm{~nm}$

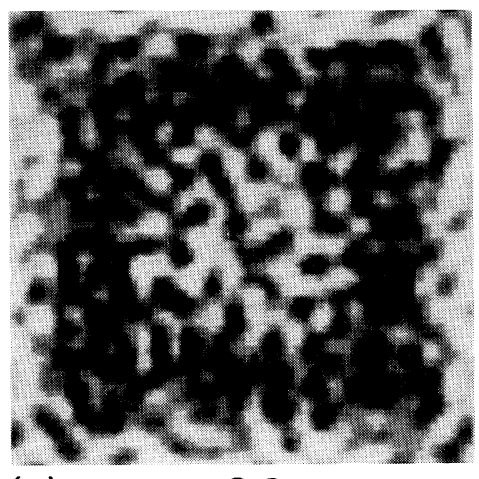

(e)

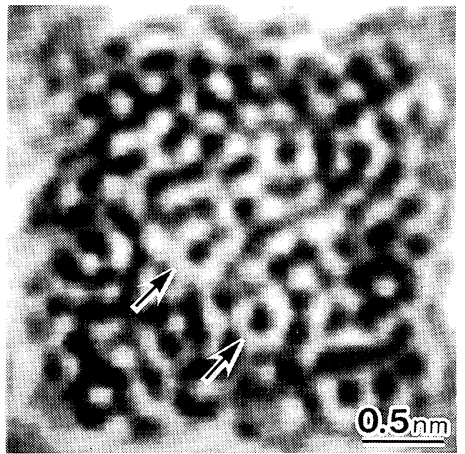

(c)

$80 \mathrm{~nm}$

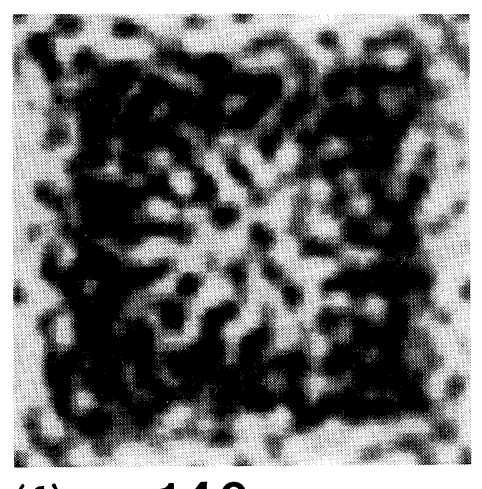

(f) $140 \mathrm{~nm}$

Fig. 1 Calculated images of $\mathrm{Pd}_{80} \mathrm{Si}_{20}$ DRP structure with 600 atoms for a series of defocus (underfocus) values: (a) 40, (b) 60, (c) 80 , (d) 100 , (e) 120 and (f) $140 \mathrm{~nm}$.

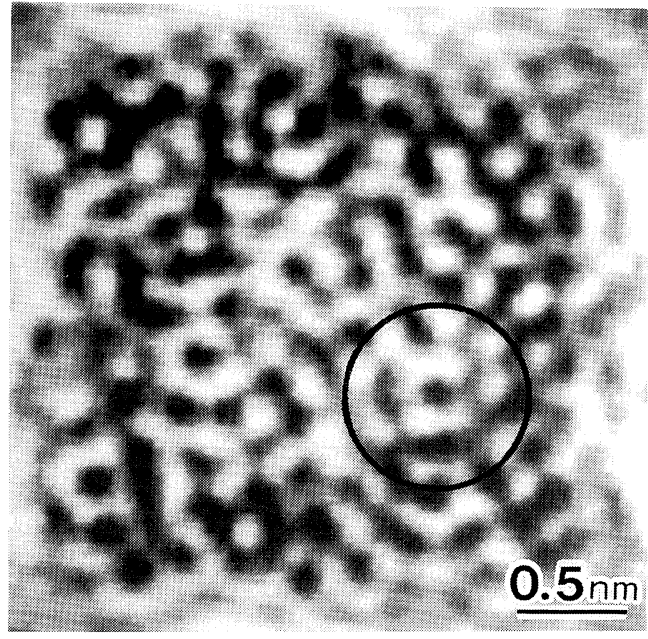

(a)

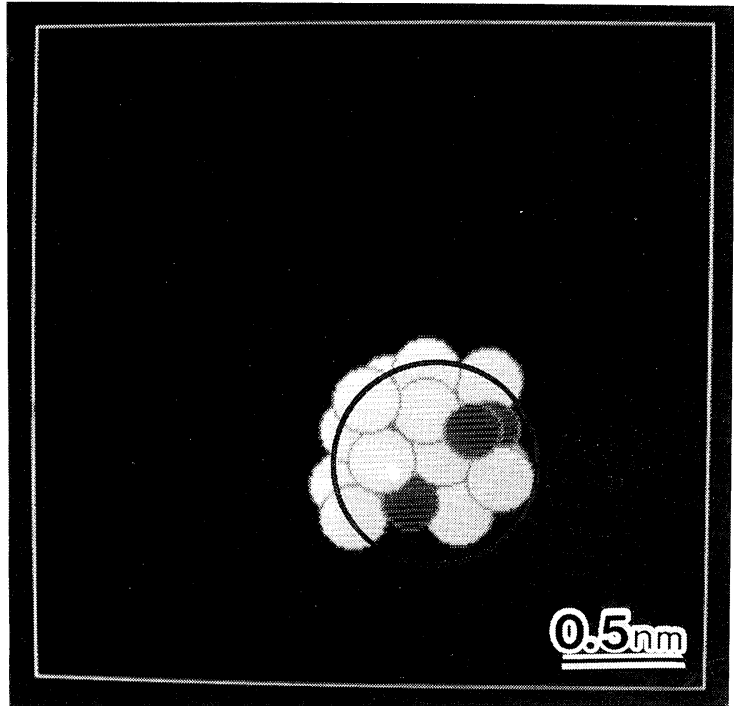

(b)

Fig. 2 (a) Pseudo-circular contrasts in the image of DRP structure, same that shown in Fig. 1(c). An example of the contrast is encircled. (b) Atomic arrangements at the region corresponding to the encircled area in (a).

structure is removed. But when the icosahedral cluster is enveloped in the DRP structure, the image changes drastically to lose its characteristic symmetry as a result of the overlapping of atomic potentials of the icosahedral structure and of the DRP structure.
However, characteristic concentric bright circles (indicated by a pair of small arrows) with an inner circle with diameter $0.26 \mathrm{~nm}$ are formed clearly around the central dark dot (indicated by a large arrow) under the defocus $\Delta f \sim 80 \mathrm{~nm}$. Through-focus image calculations 


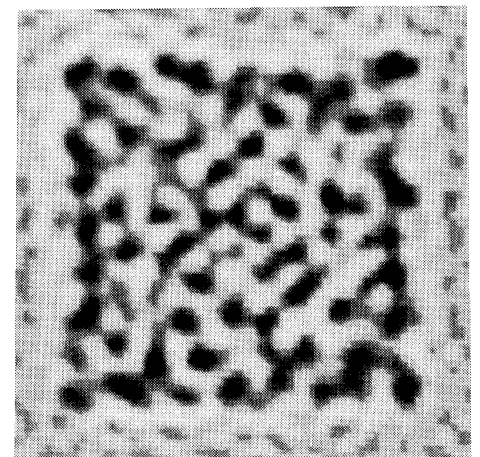

(a)

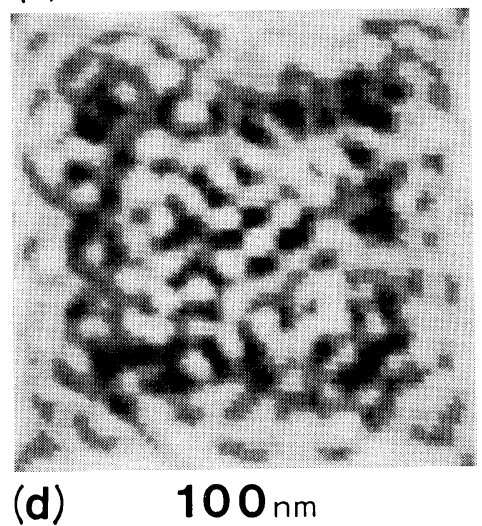

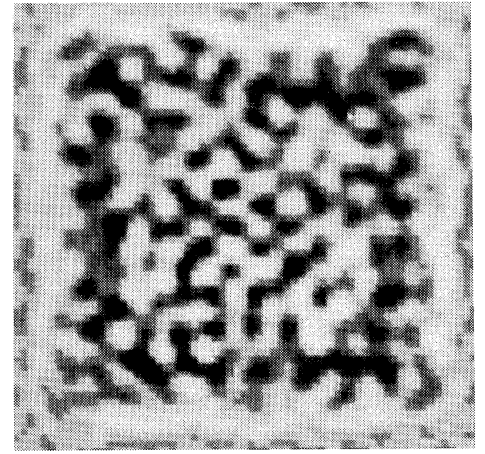

(b)

$60 \mathrm{~nm}$

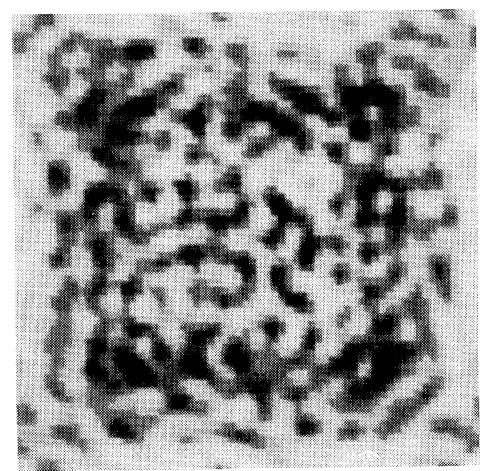

(e) $\quad 120 \mathrm{~nm}$

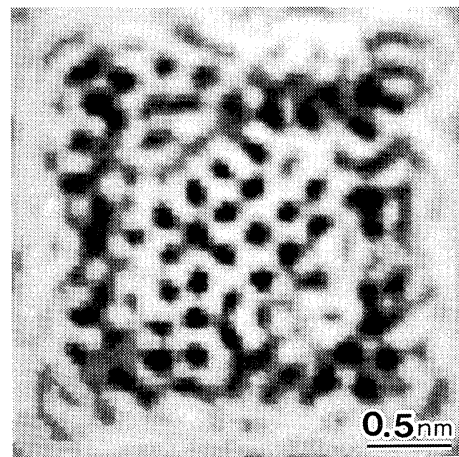

(c)

$80 \mathrm{~nm}$

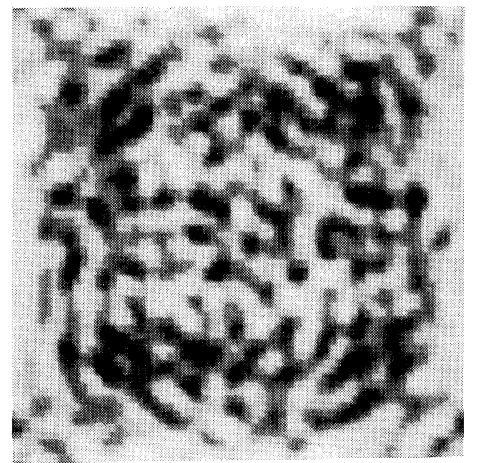

(f) $140 \mathrm{~nm}$

Fig. 3 Calculated images of $\mathrm{Pd}_{80} \mathrm{Si}_{20}$ FCC cluster with 106 atoms embedded in the $\mathrm{Pd}_{80} \mathrm{Si}_{20}$ DRP structure with 420 atoms for defocus (underfocus) values: (a) 40, (b) 60, (c) 80, (d) 100, (e) 120 and (f) $140 \mathrm{~nm}$. Beam direction is parallel to [101] of the FCC cluster. Crossed lattice fringes from the $\{111\}$ planes (spacing: $0.22 \mathrm{~nm}$ ) of the cluster are clearly observed under the defocus of $80 \mathrm{~nm}$.

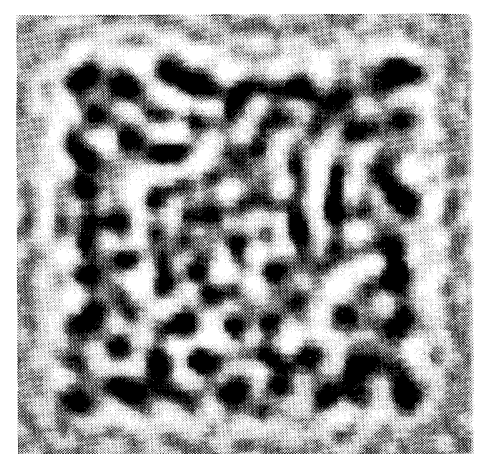

(a)

$$
40 \mathrm{~nm}
$$

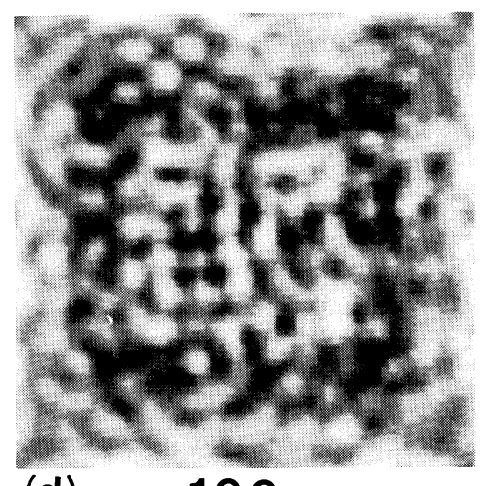

(d)

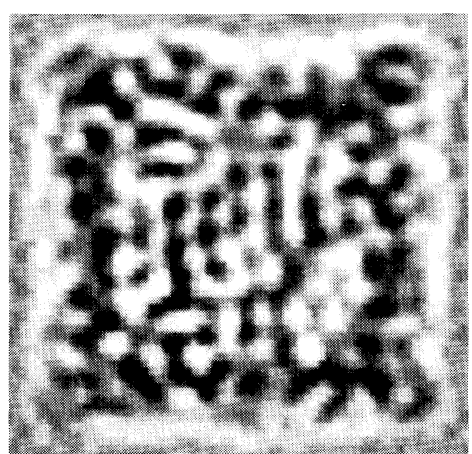

(b)

$$
60 \mathrm{~nm}
$$

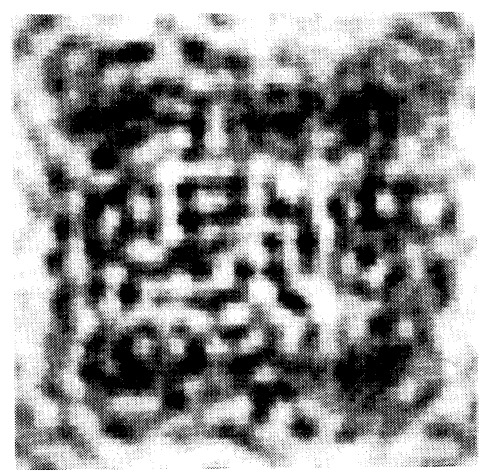

(e)

$120 \mathrm{~nm}$

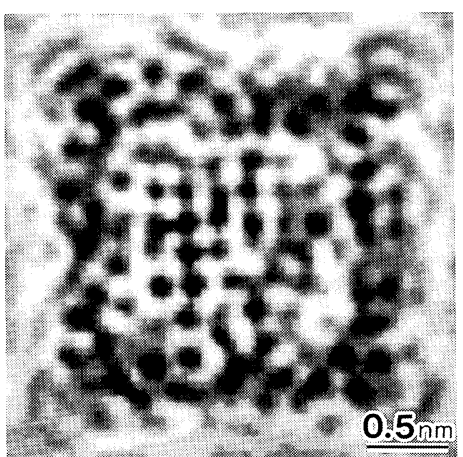

(c)

$80 \mathrm{~nm}$

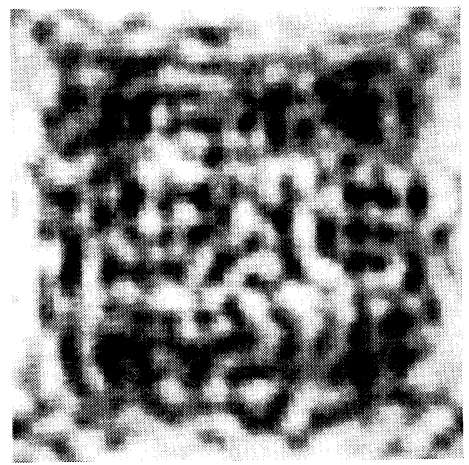

(f) $140 \mathrm{~nm}$

Fig. 4 Calculated images of $\mathrm{Pd}_{80} \mathrm{Si}_{20}$ FCC cluster with 106 atoms embedded in the $\mathrm{Pd}_{80} \mathrm{Si}_{20}$ DRP structure with 420 atoms for defocus (underfocus) values: (a) 40, (b) 60, (c) 80, (d) 100, (e) 120 and (f) $140 \mathrm{~nm}$. Beam direction is parallel to [001] of the FCC cluster. Crossed lattice fringes from the $\{200\}$ planes (spacing: $0.19 \mathrm{~nm}$ ) of the cluster are clearly observed under the defocus of $80 \mathrm{~nm}$. 


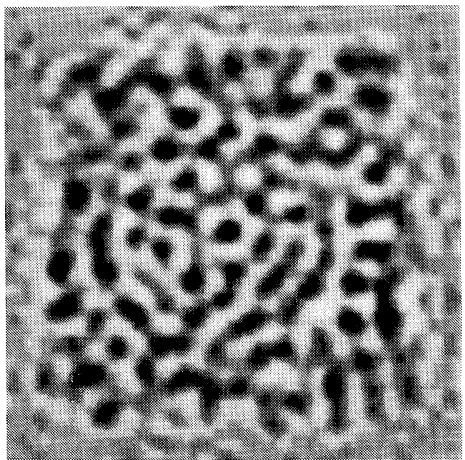

(a)

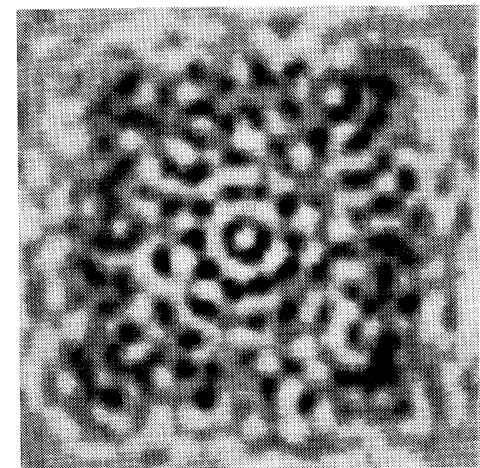

(d) $100 \mathrm{~nm}$

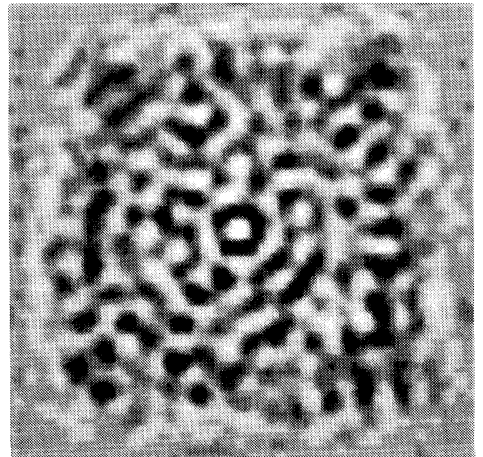

(b)

$60 \mathrm{~nm}$

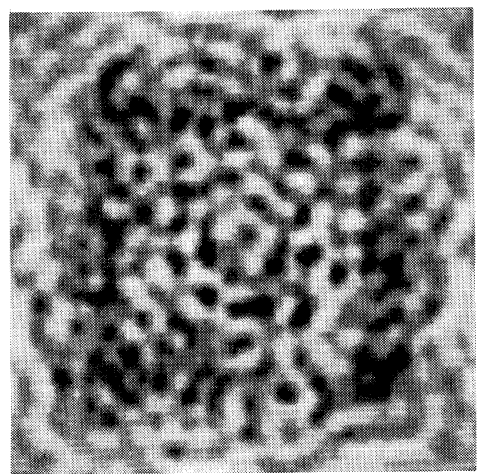

(e)

$120 \mathrm{~nm}$

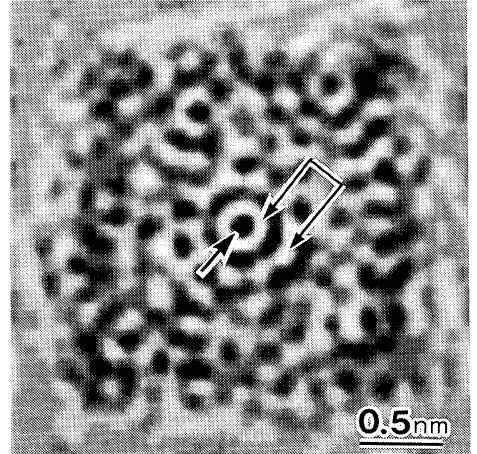

(c) $80 \mathrm{~nm}$

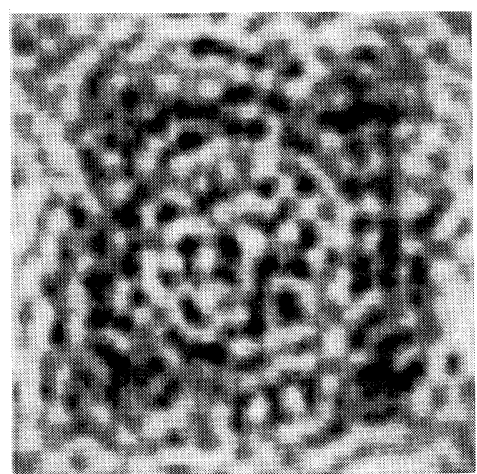

(f) $140 \mathrm{~nm}$

Fig. 5 Calculated images of icosahedral $\mathrm{Pd}_{80} \mathrm{Si}_{20}$ cluster with 147 atoms embedded in the $\mathrm{Pd}_{80} \mathrm{Si}_{20}$ DRP structure with 420 atoms for defocus (underfocus) values: (a) 40, (b) 60, (c) 80, (d) 100, (e) 120 and (f) $140 \mathrm{~nm}$. Beam direction is parallel to the five-fold axis of the icosahedron.

were also made along the three and two-fold axes of icosahedron. Along the two-fold axis direction, twinned bright concentric circular images are produced symmetrically around the two-fold axis under the defocus $\Delta f \sim 80 \mathrm{~nm}$. The image along the three-fold axis does not give any characteristic contrast under various defocus conditions. Since there are several five-fold and two-fold axes in the icosahedron ( 6 and 15, respectively), a frequency of finding such bright concentric circular contrasts may be quite large, if the icosahedral clusters were formed in the alloys. It was found that the arrangement of straight atomic chains parallel to the incident beam direction is essentially important to form a high contrast image of MRO domains embedded in the DRP structure. Such a geometrical situation is satisfied in the case of microcrystalline MRO domains but is not in the icosahedral atom cluster.

\section{Experimental}

Amorphous $\mathrm{Pd}_{80} \mathrm{Si}_{20}$ thin films were made by an $\mathrm{Ar}$ beam sputtering technique with a newly assembled beam sputtering apparatus equipped with an water-cooled beam-gun (Ion-Tech. FAB-110, $2 \mathrm{kV}-50 \mathrm{~mA}$ ) suppling neutral Ar beam with a diameter of about $1 \mathrm{~cm}$. A $\mathrm{Pd}_{80} \mathrm{Si}_{20}$ alloy was used as a sputtering target and after evacuation to $1.3 \times 10^{-5} \mathrm{~Pa}$ the deposition was made onto $\mathrm{NaCl}$ substrates cooled down to $195 \mathrm{~K}$ by liquid nitrogen. The film thickness was about $10 \mathrm{~nm}$ which was estimated from a deposition time-thickness curve for this alloy. The sputtered films were observed under the axialbeam illumination condition in a high resolution electron microscope JEM-2010 operating at $200 \mathrm{kV}$. The amount of defocus was estimated from the focal steps of the objective lens-current knob.

\section{Results and Discussion}

\section{MRO structure of the $\mathbf{P d}_{80} \mathrm{Si}_{20}$ alloy}

According to the image calculations, the most suitable defocus conditions for the observations of FCC and icosahedral atom clusters are almost in the same range. The HREM observation of the $\mathrm{Pd}_{80} \mathrm{Si}_{20}$ thin film was, therefore, made under the defocus near $\Delta f \sim 80 \mathrm{~nm}$ (underfocus). Figure 6 is a high resolution image taken under the defocus near $80 \mathrm{~nm}$. MRO domains with crossed lattice images are locally observed and from the geometries of their images the MRO domains is found to have FCC structure extending from 1 to $2 \mathrm{~nm}$ in size. Examples of such images are shown in the encircled areas in the micrograph. As expected from the image calculation in section II, shapes of the FCC clusters are not imaged.

In the micrograph, bright pseudo-circular images with a diameter near $0.23 \mathrm{~nm}$ and a little larger size are frequently observed as indicated by single and double ar- 


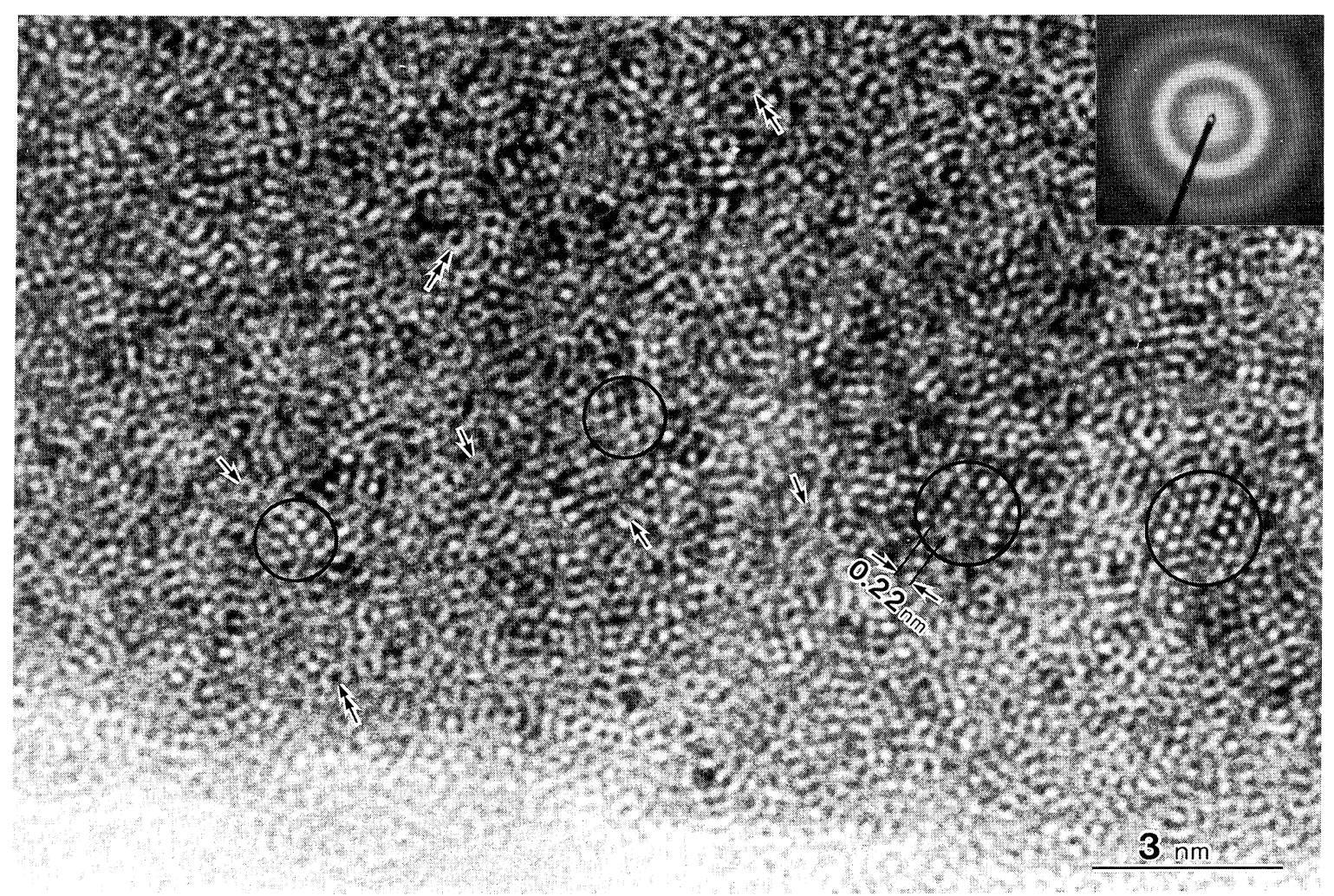

Fig. 6 HREM image of sputter-deposited amorphous $\mathrm{Pd}_{80} \mathrm{Si}_{20}$ thin film. A corresponding electron diffraction pattern is also shown. The FCC MRO regions are seen in the encircled areas. Pseudo-circular bright contrasts are seen indicated by arrows.

rows, respectively. By referring to the contrasts in the calculated images of the DRP structure where such pseudo-circular bright contrasts are often seen, these images are thought to come mostly from the DRP structure. Since concentric bright circular contrasts as shown in Fig. 5(c) having clear inner bright circles with diameter of $0.26 \mathrm{~nm}$ are not observed, it can be said that there is no positive evidence of the formation of icosahedral atomclusters in the sputter-deposited Pd-Si alloys. Other possible reason for the appearance of such contrasts will be discussed later.

According to Hamada and Fujita ${ }^{(13)}$, lattice fringe images from BCC atom clusters in the DRP structure are expected to appear in amorphous $\mathrm{Fe}-\mathrm{B}$ alloys under a wide range of incident beam directions. In the present study, the incident beam directions to the FCC cluster (106 atoms) were varied stepwise from [001] to [101] and [111], from [101] to [111], and from [001] to [313], [212] and [434] directions, and for each incidence the image of the cluster was calculated. Figure 7 shows a stereographic projection for the cubic crystal, where the dotted regions cover the incident beam directions to give crossed-fringes (two-dimensional lattice images) and the shaded regions cover those to show parallel fringes (one-dimensional lattice images) from the FCC cluster. It is seen that the lattice fringes can be observed from the wide range of the incident beam directions in the case of small FCC cluster. The coverage of the angle of incident beam direction which contributes to the lattice imaging of the cluster can be estimated from the stereograph as $60 \%$. One and two dimensional lattice fringes, therefore, must appear very frequently, suppose that the deposited thin film is composed only of the FCC clusters. In the obtained image, however, such lattice fringe images were not so frequently observed, meaning that the DRP-like structure regions occupy a large volume fraction.

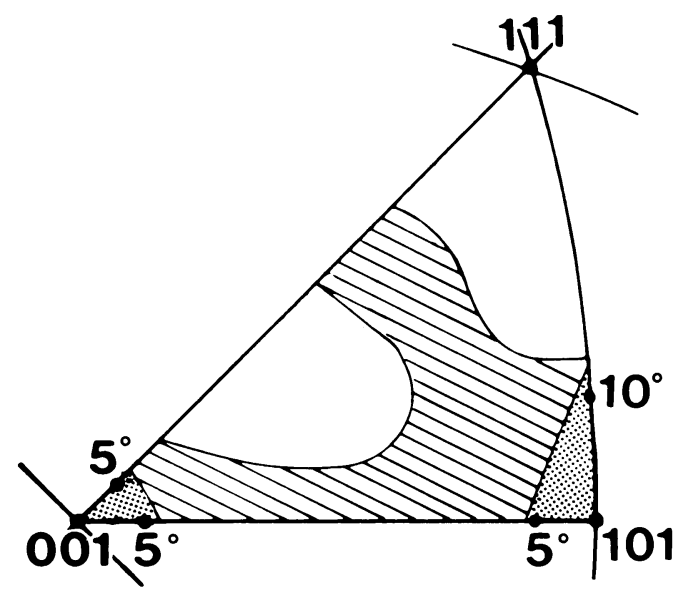

Fig. 7 Range of beam incident direction to give $\{111\}$ or $\{200\}$ lattice fringes in the HREM images shown in the stereo-triangle of cubic crystal system with the [001] pole. Dotted area indicates a range of the beam incidence to give crossed lattice fringes and shaded area, parallel fringes. 


\section{Comparison with HREM images of $\mathrm{Pd}-\mathrm{Cu}-\mathrm{Si}$ alloy}

$\mathrm{Pd}_{77.5} \mathrm{Cu}_{6} \mathrm{Si}_{16.5}$ is known to have a very high glass-forming ability. In the previous structural studies on the rapidly-quenched $\mathrm{Pd}_{77.5} \mathrm{Cu}_{6} \mathrm{Si}_{16.5}$ alloy ribbons ${ }^{(3)(4)}$, lattice fringe images from MRO structures with FCC domains were frequently observed extending in a range from 1 to $2 \mathrm{~nm}$, in spite of the appearance of typical diffuse halo rings in diffraction patterns. The average size and the volume fraction of these domains were found to change as a function of the rapid-quenching condition. Figure $8(a)$ and (b) are examples of the HREM images ${ }^{(5)}$ taken from thin areas of the specimens rapidly-quenched under the quenching rates (estimated from the thermo-transfer equation ${ }^{(3)}$ ) of about $6 \times 10^{5} \mathrm{~K} / \mathrm{s}$ and $4 \times 10^{6} \mathrm{~K} / \mathrm{s}$, respectively. These images were taken under the most appropriate defocus condition (near $160 \mathrm{~nm}$ ) for an electron microscope with $\mathrm{Cs}=1.2 \mathrm{~mm}$. Lattice fringe images of FCC domains are frequently observed in Fig. 8(a), while they are not observed so often in Fig. 8(b) but DRP-like structure similar to that in Fig. 6 is observed. It is said that the structure of amorphous alloy ribbons liquid-quenched under a high quenching rate (of the order of $10^{6} \mathrm{~K} / \mathrm{s}$ ) must be quite similar to that of the amorphous alloys formed by the vapor-quenching technique. On the other hand, the structure of amorphous alloys formed under the quenching rate of the order of $10^{5} \mathrm{~K} / \mathrm{s}$ or oless must be composed of dense FCC clusters. Considerable displacements of atoms in the FCC clusters from ideal atomic positions must be realized due to the
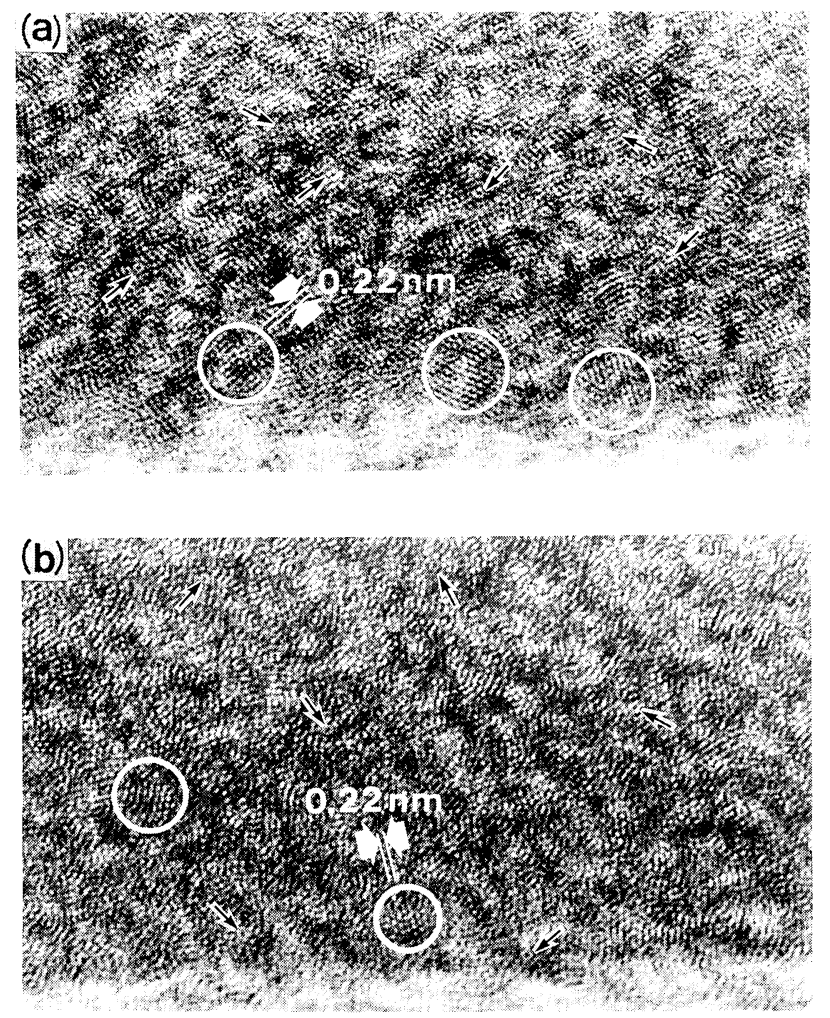

Fig. 8 HREM images taken from the amorphous $\mathrm{Pd}_{77.5} \mathrm{Cu}_{6} \mathrm{Si}_{16.5}$ alloy ribbons with quenching rates (a) $\sim 6 \times 10^{5} \mathrm{~K} / \mathrm{s}$ and (b) $\sim 4 \times 10^{6} \mathrm{~K} / \mathrm{s}$. supersaturated solution of the non-metal atoms and also due to the structural relaxation in relation to the enveloping structure, and these displacements are thought to contribute to the diffuse-halo scattering in diffraction. In Fig. 8(a) and (b), bright pseudo-circular contrasts are also observed (indicated by arrows) as in Fig. 6.

\section{Bright pseudo-circular contrasts}

In the image calculation of the FCC atom cluster, it was found that DRP-like images appear when the incident beam direction comes near the [111] direction. Figure 9 shows a series of calculated images for the $\mathrm{Pd}-\mathrm{Si}$ FCC cluster (106 atoms) under the different incident beam directions tilted from [101] to [111]. Near and at the [111] incidences, the images change into ones similar to those from the DRP structure (see Fig. 1, for example), and bright pseudo-circular contrasts with a diameter close to $0.23 \mathrm{~nm}$ appear in these images. In Fig. 8(a), the bright pseudo-circular contrasts are seen among the FCC clusters. Because of the large volume fraction of the FCC clusters in the specimen, most of these contrasts must be due to the misoriented FCC clusters. But in the case of the deposited films, a volume fraction of the DRP-like region must be large as explained in IV-1. Therefore, only a part of the bright pseudo-circular contrasts in Fig. 6 may be responsible for the FCC clusters.

We could not observe clear concentric bright circular contrasts typical of the icosahedral atomic arrangements. However, more detailed high resolution work is necessary, especially for the regions showing the bright pseudo-circular contrasts with diameters as large as $0.26 \mathrm{~nm}$.

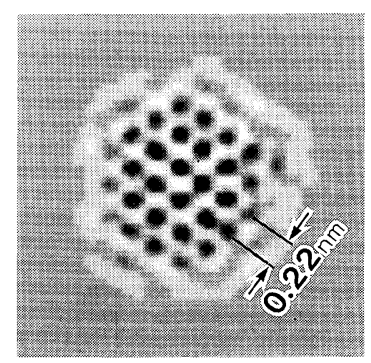

5 deg

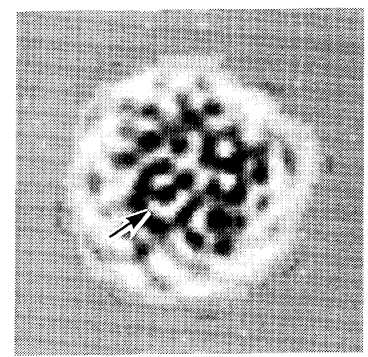

$25 \mathrm{deg}$

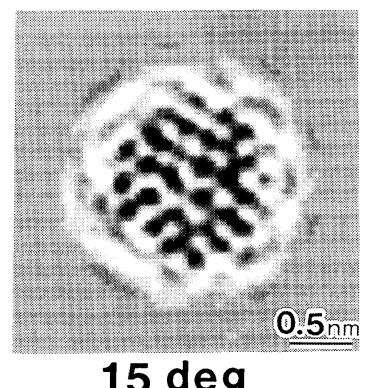

$15 \mathrm{deg}$

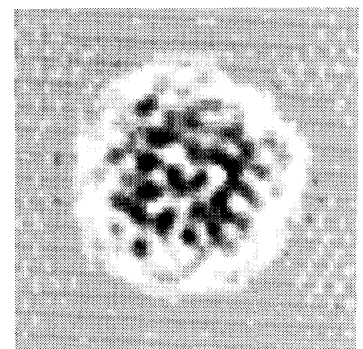

35 deg
Fig. 9 A series of calculated images of the FCC cluster with different beam incident directions from [101] towards [111] of the cluster. The amount of angle shown for each image is a deviation angle from the [101] towards [111] axis. In the DRP-like image, pseudo-circular bright contrast is seen (indicated by arrow). 


\section{RDF for the MRO structure}

From the comparison of observed images with calculated ones, FCC MRO structures are found to be preferentially formed in the near-eutectic amorphous $\mathrm{Pd}-(\mathrm{Cu})-\mathrm{Si}$ alloys. In order to explain experimental results all consistently, it is needed to show that electron or X-ray scattering from amorphous structures with MRO form the halo-diffraction pattern. In other words, the RDF profile from the FCC MRO structure model must be the same as that obtained experimentally by $\mathrm{X}$ -
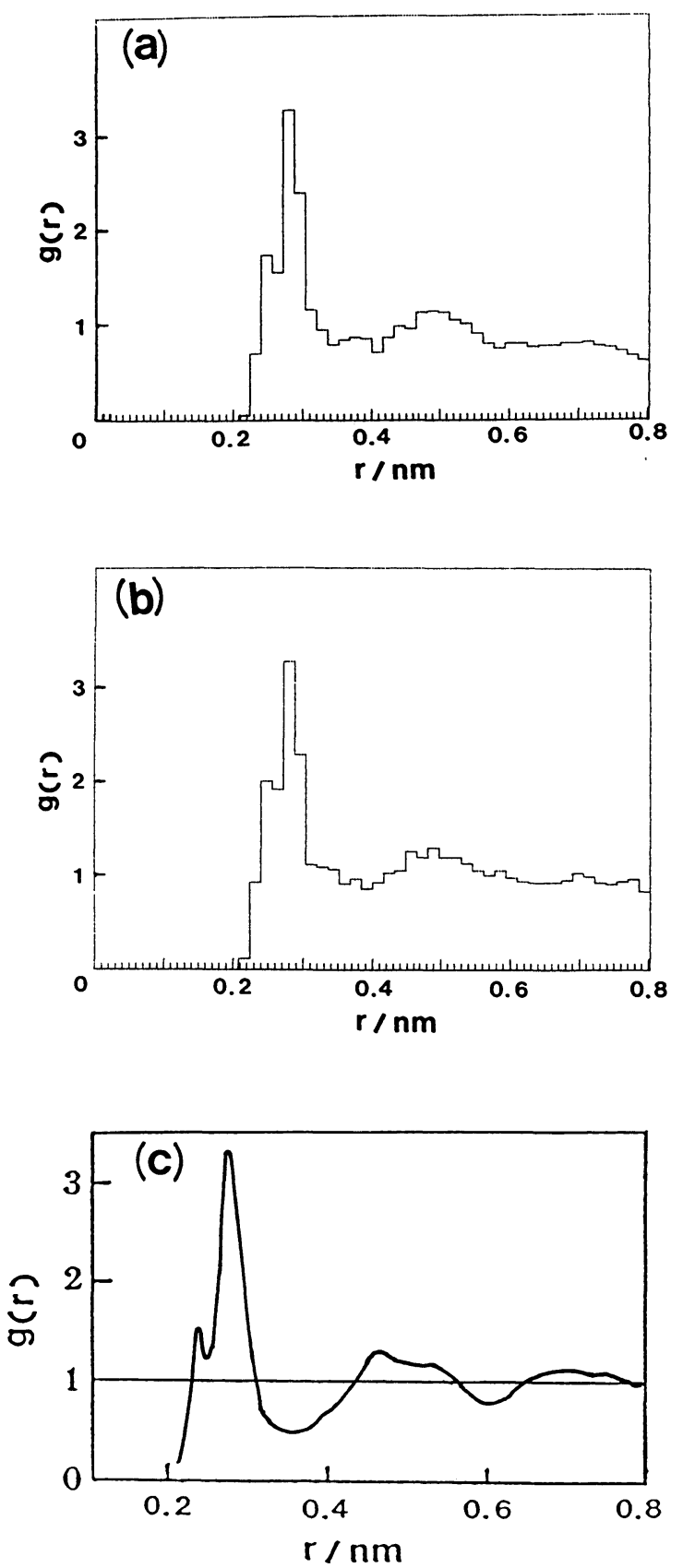

Fig. 10 (a) RDF calculated for the DRP structure composed of 600 atoms with $\mathrm{Pd}_{80} \mathrm{Si}_{20}$ composition. (b) RDF calculated for the FCC MRO structure with $\mathrm{Pd}_{80} \mathrm{Si}_{20}$ composition composed of a FCC cluster with 106 atoms and an enveloping DRP structure with 420 atoms. (c) RDF for an amorphous $\mathrm{Pd}_{80} \mathrm{Si}_{20}$ alloy experimentally obtained by Fukunaga and Suzuki ${ }^{(14)}$. ray or neutron diffraction.

Figure 10(a) shows a RDF of the DRP structure made from 600 atoms. The RDF function is found to reproduce well the experimental function Fig. 10(c) obtained from an amorphous $\mathrm{Pd}_{80} \mathrm{Si}_{20}$ alloy by Fukunaga and Suzuki ${ }^{(14)}$. On the other hand, Fig. 10(b) shows a RDF for the FCC MRO structure with a composition $\mathrm{Pd}_{80} \mathrm{Si}_{20}$ in which a FCC cluster with 106 atoms is embedded in 420 DRP atoms. By comparing Fig. 10(a) and 10(b) with Fig. 10(c), it is presumed that not only the DRP structure but also the MRO structure can explain the experimental intensity profiles of X-ray or neutron diffraction from amorphous alloys in general, as pointed out by Hamada and Fujita ${ }^{(7)}$ in their study of RDF calculation for their MRO model in amorphous Fe-B alloy.

\section{Summary and Conclusion}

HREM images of the amorphous structure of Pd-Si alloy with near-eutectic composition have been calculated based on the structure models of DRP, MRO with FCC atom cluster and MRO with icosahedral atom cluster. These images were compared with those observed in a $\mathrm{Pd}_{80} \mathrm{Si}_{20}$ alloy prepared by a sputter-deposition technique. The amorphous structures of the present alloy films and those of the $\mathrm{Pd}_{77.5} \mathrm{Cu}_{6} \mathrm{Si}_{16.5}$ alloy ribbons observed previously were compared an the differences were discussed. The findings of this study are summarized as follows.

(1) MRO domains with FCC atom clusters were observed in the sputter-deposited amorphous $\mathrm{Pd}-\mathrm{Si}$ alloy films. The volume fraction of such areas is small compared with that in the amorphous $\mathrm{Pd}-\mathrm{Cu}-\mathrm{Si}$ ribbons formed under the cooling rate near $10^{5} \mathrm{~K} / \mathrm{s}$, but is almost comparable with that in the ribbons formed under the cooling rate near $10^{6} \mathrm{~K} / \mathrm{s}$.

(2) Because of the similarity between the observed and the calculated images, most of the structure enveloping the FCC clusters is thought to be the DRP structure in the Pd-Si alloy films.

(3) As a characteristic feature of the DRP structure, pseudo-circular bright contrasts with a diameter near $0.23 \mathrm{~nm}$ were observed frequently. The image calculation showed that a ring-like atomic arrangement with five or six-fold symmetry around a central atom is responsible for these contrasts.

(4) The FCC clusters oriented far away from the $\langle 100\rangle$ or $\langle 110\rangle$ zone axis give DRP-like images. However, in the case of FCC clusters, a chance of finding such DRP-like images is small compared with that of finding $\{111\}$ or $\{200\}$ lattice fringes.

(5) Calculated RDF for the FCC MRO model can explain the RDF obtained from a X-ray diffraction study by Fukunaga and Suzuki.

\section{Acknowledgements}

The authors would like to express their thanks to Professor S. Nagakura for his helpful discussion and en- 
couragement. This research was partly supported by the Grant-in-Aid for Fundamental Researches from the Ministry of Education, Science and Culture, Japan and also by the Grant from Shinsei Shigen Kyokai, Japan.

\section{REFERENCES}

(1) P. H. Gaskell, D. J. Smith, C. J. D. Catto and J. R. A. Cleaver: Nature, 281 (1979), 465.

(2) H. Ichinose and Y. Ishida: Trans. Japan Inst. Metals, 24 (1983), 405.

(3) Y. Hirotsu and R. Akada: Jpn. J. Appl. Phys., 23 (1984), L479.

(4) Y. Hirotsu, M. Uehara and M. Ueno: J. Appl. Phys., 59 (1986), 3081.
(5) Y. Hirotsu and M. Uehara: J. Japan Inst. Metals, 52 (1988), 129.

(6) J. C. Phillips: J. Non-Cryst. Solids, 43 (1981), 37.

(7) T. Hamada and F. E. Fujita: Jpn. J. Appl. Phys., 21 (1982), 981.

(8) R. Yamamoto and M. Doyama: J. Phys., F 9 (1979), 617.

(9) P. J. Steinhardt, D. R. Nelson and M. Ronchetti: Phys. Rev., B 28 (1983), 784.

(10) S. Nose and F. Yonezawa: J. Chem. Phys., 84 (1986), 1803.

(11) T. Sakata, H. Fujita and N. Sumida: Proc. XIth Int. Cong. on Electron Microscopy, Kyoto, (1986), p. 1545.

(12) J. M. Dubois, P. H. Gaskell and G. Le Caer: Proc. Roy. Soc. Lond., A402 (1985), 323.

(13) T. Hamada and F. E. Fujita: Jpn. J. Appl. Phys., 25 (1986), 318

(14) T. Fukunaga and K. Suzuki: Sci. Rep. Res. Inst. Tohoku Univ., A29 (1981), 153 